Management Accounting \& Control Scales Handbook

Schäffer, Utz: Management Accounting \& Control Scales Handbook, 1. Aufl., Wiesbaden: Gabler, 2007, 310 Seiten, CHF 85,00/ EUR (D) 49,90, ISBN 978-3-8350-0525-9

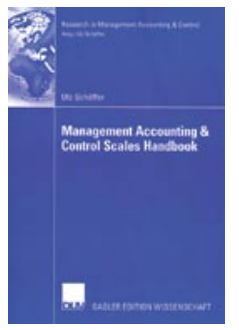

Die erste Auflage von Utz Schäffers Management Accounting \& Control Scales Handbook ist ein neues umfassendes Nachschlagewerk für quantitative Forschung in den Bereichen internes Rechnungswesen und Controlling.

Das Buch gibt einen exzellenten Überblick über die neuesten entwickelten Konstrukte im Controlling. Dadurch wird den Forschenden die mühsame Suche oder die eigene Entwicklung und Validierung von Konstrukten erspart. Dieses Buch ermöglicht Forschern auf dem Gebiet des Controlling oder anderen Fachrichtungen die praktische Umsetzung einer geeigneten Controllingskala. Forschern, welche sich zuvor weniger mit Themen des internen Rechnungswesens auseinandersetzten, wird überdies der Zugang zu den vielfältigen Konstrukten des Controlling bedeutend erleichtert.

Utz Schäffers Sammelband beinhaltet zahlreiche interessante Konstrukte aus unterschiedlichen Controllingbereichen wie beispielsweise Budgetierung, Verhaltenssteuerung und symbolische Nutzung von Informationen. Die Konstrukte werden nach einem standardisierten Schema einzeln vorgestellt. Der Autor beschreibt sie kurz, nennt ihre Herkunft und Einsatzweise und empfiehlt anschließend weiterführende Literatur.

Viele der in diesem Buch vorgestellten Konstrukte wurden allerdings in der deutschsprachigen Literatur entwickelt. Für Forscher, welche Veröffentlichungen in englischsprachigen Journals anstreben, bedeutet dies, dass sie die einzelnen Items übersetzen und anschließend die Validität der Konstrukte neu überprüfen müssen. Um die Anwendbarkeit im englischsprachigen Raum zu erhöhen, wäre eine solche Validierung der im deutschsprachigen Raum entwickelten Konstrukte im Rahmen einer zweiten Auflage wünschenswert.

Dennoch bleibt festzuhalten, dass Utz Schäffer mit seinem Managing Accounting \& Control Scales Handbook einen bedeutenden Beitrag zur Forschung im Bereich Controlling leistet. Forschende, welche primär im Bereich Controlling oder an deren Schnittstellen wie beispielsweise Marketingcontrolling oder Logistikcontrolling arbeiten, erhalten eine hervorragende Übersicht über die vielfältigen in diesem Gebiet verwendeten Konstrukte.

Aufgrund des sehr wissenschaftlichen Charakters ist das Buch für den Praktiker hingegen weniger empfehlenswert.

V.S. Schröder

\section{In-Game Advertising}

Thomas, Wolfgang/Stammermann, Ludger: In-Game Advertising - Werbung in Computerspielen: Strategien und Konzepte, Wiesbaden: Gabler, 2007, 159 Seiten, CHF 70.00/ EUR (D) 42.00, ISBN 978-3-8349-0702-8

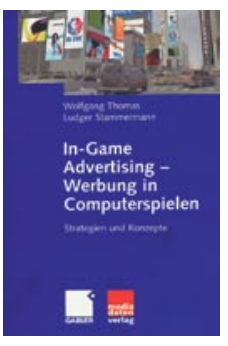

Auf der Suche nach einem neuen Zugang zur werberelevanten „Spieler-Zielgruppe“ der 20bis 40-Jährigen hat die Werbebranche in den vergangenen Jahren zunehmend die Werbung in Computerspielen entdeckt. Das Buch von Thomas und Stammermann erklärt die neuesten Entwicklungen des digitalen Werbemarktes und zeigt auf, welchen Regeln er unterliegt. Von den Grundlagen und Praxisanwendungen über unterschiedliche Erfolgsbeispiele bis hin zur Zukunftsvision werden alle relevanten Themengebiete des In-Game Advertising betrachtet. Die Erkenntnisse der beiden Medienforscher basieren auf Studien und einer aktuellen Umfrage unter mehr als 100 Marketingentscheidern. Das Ziel dieses Buches ist es, diese werberelevante Zielgruppe für Marketingentscheider zugänglich zu machen, wobei In-Game Advertising bei der Mediaplanung und im Media-Mix berücksichtigt wird.

Die einzelnen Kapitel sind anwenderorientiert geschrieben und geben ein breites
Abbild der betrachteten Fragestellung. Der Einblick in die aktuelle Literatur ist knapp gehalten. Da es sich allerdings um eine aktuelle Thematik handelt, spielt dieser Aspekt eine eher untergeordnete Rolle. Mit Hilfe des beigefügten Glossars kann eine Vielzahl von Stichworten nachgeschlagen werden. Als Ergänzung zum Buch werden auf einer Internetseite Informationen zu aktuellen Entwicklungen im Media-Marketing kostenlos bereitgestellt.

Damit ist sichergestellt, dass in der schnelllebigen digitalen Werbewelt neuere Entwicklungen des In-Game Advertising Berücksichtigung finden.

Die Forschung zur Nutzung von Computerspielen zu Werbezwecken rückt durch die aktuellen Entwicklungen auf dem Onund Offline-Spielemarkt immer mehr in den Fokus der internationalen Unternehmen. Dieses Buch richtet sich vornehmlich an Praktiker, vermittelt aber auch interessierten Studierenden auf der Master-Stufe als auch Forschern im Bereich Media-Marketing erste Grundlagen sowie interessante „Best Practice“-Beispiele.

V. S. Schröder 